\title{
BIOGEOGRAPHIC AND EVOLUTIONARY CONTROLS ON THE MARINE LATITUDINAL DIVERSITY GRADIENT
}

ROY*, Kaustuv, Department of Biology 0116, University of California, San Diego, La Jolla, CA 92093-0116; JABLONSKI, David, Department of Geophysical Sciences, University of Chicago, 5734 S. Ellis Avenue, Chicago, IL 60637; VALENTINE, James W., Museum of Paleontology, University of California, Berkeley, CA 94720.

Latitudinal diversity gradients characterize many marine and terrestrial groups, and despite a long debate about the factors that produce them, they remain enigmatic. Analyses of distributional patterns of shallow marine mollusks living on the eastern Pacific shelf contradict some current theories about the controls on such gradients, and reveal the important role played by biogeographic barriers. The Pleistocene fossil record along this coast shows the existence of such biogeographic controls in the past, and the Neogene fossil record of eastern Pacific mollusks strongly suggests that an evolutionary dynamic underlies the tropical-temperate gradient, with tropical faunas having higher turnover rates.

The eastern Pacific mollusks show a strong latitudinal diversity gradient from the equator to northern Alaska; the diversity gradient has a stepped pattern with the major changes in diversity concentrated at provincial boundaries. These boundaries are correlated with contacts between contrasting water masses or water types with distinctive temperature regimes and hence provide physical barriers to species migration. The shape of the diversity gradient is thus controlled by the spacing of these oceanographic barriers. In addition, the barriers are perceived differently by northern and southern species. For southern species the provincial boundaries represent cold water barriers that can only be crossed by very shallow and physiologically tolerant species; in contrast, for northern species these boundaries represent warm water barriers that are leaky filters since cold water can be found in deeper environments. Thus, $31 \% \pm$ $2 \%$ of all northern range endpoints cluster at the provincial boundaries compared to only $17 \% \pm 2 \%$ of the southern range endpoints. Pleistocene data indicate that this asymmetry of barrier effectiveness has been present over geologic time, and strongly influences the responses of species to climatic fluctuations. Southern species that crossed provincial boundaries in response to climatic change tend to be significantly longer ranging and bathymetrically shallow compared to their parent species pool while the northern species exhibiting such movement are drawn randomly from the available species pool.

While provinciality produces an important second order control on the shape of the latitudinal diversity gradient, the primary controls are still unclear; one major source of uncertainty is the paucity of direct evidence on origination and extinction patterns . Neogene molluscan faunas along the eastern Pacific indicate that latitudinal gradients in turnover rates do indeed exist. Lyellian Percentages (L.P.) calculated for a number of Neogene molluscan assemblages show a tropical-temperate gradient; L.P.s for Mid Miocene to Recent tropical assemblages are significantly lower than similar age temperate and high latitude faunas. Analysis of Neogene molluscan faunas of the western Pacific also reveals a similar gradient in turnover rates suggesting that the pattern may be a general one.

Thus analyses of present day distributional patterns of eastern Pacific marine mollusks in conjunction with paleobiological data reveal a dynamic picture of biodiversity where evolutionary rate differences underlie the primary diversity gradient. However, the exact nature of this gradient is controlled by spatial distribution of biogeographic boundaries that are determined by prevailing physical conditions. 